\title{
Classification of Simultaneous Motions using Wavelet-based Time-frequency Representation of EMG Signals
}

\author{
Nan $\mathrm{Bu}^{\mathrm{a}, *}$, Takayuki Mukaeda ${ }^{\mathrm{b}}$ \\ ${ }^{a}$ National Institute of Technology, Kumamoto College, 2659-2 Suya, Koshi, 861-1102, JAPAN \\ bYokohama National University, 79-5 Tokiwadai, Hodogaya, Yokohama, 240-8501, JAPAN \\ *Corresponding Author: bu@kumamoto-nct.ac.jp
}

\begin{abstract}
The aim of our study is to develop a pattern recognition method to classify simultaneous motions from surface electromyographic (EMG) signals, with which a multi-degrees of freedom (DOFs) myoelectric control interface can be realized. Wavelet transform has been adopted in order to extract time-frequency (TF) feature patterns from EMG signals. However, an essential problem is that the TF feature vectors usually occupy a high-dimension space, which may cause degradation in classification performance.

To deal with this problem, this paper applied a sub-band feature reduction method to the wavelet-based TF features. This method divides the frequency bandwidth into several sub-bands, and defines feature patterns on each sub-band to replace the original feature vectors. Then, the dimension-reduced feature patterns are sent to a multi-layer perceptron (MLP) to conduct motion classification.

Experiments were carried out with four subjects to verify the proposed method. In this study, eleven forearm motions, including seven single motions and four simultaneous motions, were examined with EMG signals measured from six channels. In addition, the number of sub-bands was investigated. The experimental results indicated that, except cases of six sub-bands frequency division, the average overall classification rates exceeded $90 \%$. Relatively high classification performance was achieved.
\end{abstract}

Keywords: Electromyography (EMG), time-frequency features, motion classification, dimensionality reduction, simultaneous motions.

\section{Introduction}

Electromyographic (EMG) signals, accompanied by body movements, provide a wide scope of information for motion analysis, such as force level and timing of muscular contractions. A variety of research studies have been carried out to develop EMG-based motion classification methods for applications of rehabilitation and robotic control ${ }^{(1)}$. Recent advances in device hardware have led to significant developments of powered limb prostheses and multi-articulated prosthetic hands. Improvement is still required in order to perform simultaneous movements of multiple joints toward increase of multiple degrees of freedom (DOFs) operability and realization of a high level of dexterity ${ }^{(2-5)}$.

Motion classification is an attractive field in EMG signal processing. A set of distinguishing features is extracted from EMG signals and then applied to a classifier to identify intended motions from a variety of predefined classes of motions. Control commands can then be generated with the classified motions. Most of the state of the art motion classification methods are limited to classify one single motion (usually corresponding to one DOF) in one decision, leading to an essentially sequential myoelectric control scheme.

However, natural movements are usually simultaneous activations of multiple DOFs. In the past decade, attempts have been made to classify simultaneous movements from EMG signals based on combination and/or analysis of outputs from traditional classifiers for single motions. For example, Tsuji et al. introduced a muscle synergy theory into a traditional motion classification $\operatorname{method}^{(2)}$. Simultaneous motions (e.g. wrist flexion during hand 
grasping) can be expressed by combinations of synergy patterns of EMG signals, which may be classified as single motions. In addition, a conditional parallel strategy has been developed to classify combined movements ${ }^{(4)}$. A parallel set of traditional linear discriminant analysis (LDA) classifiers is used for each discrete motion, and then outputs of these discrete motions are analyzed to generate a final motion output.

Alternatively, simultaneous motion classification may rely much more on effective feature representations extracted from EMG signals. EMG signals are inherently non-stationary. Features of time-frequency (TF) domain have been extensively studied in order to use time-varying characteristics of EMG signals for motion classification. Wavelet transform (WT) is a popular TF analysis method for non-stationary signals; wavelet-based TF feature representation has already extracted increasing attentions. It enables localized transient analysis of EMG signals. Englehart et al. utilized TF features, which were extracted with short-time Fourier transform (STFT), WT, and wavelet packet transform (WPT), to classify single motions using LDA and MLP classifiers ${ }^{(6)}$. Many other research studies indicated that WT is useful for EMG signal processing, especially for dynamic movements ${ }^{(7)}$.

In this study, continuous WT is used to generate EMG $\mathrm{TF}$ representation for simultaneous motion classification. However, an essential problem of $\mathrm{TF}$ features is that the feature vectors usually occupy a high-dimension space, since the feature dimension is determined according to the number of EMG channels and the resolution in the frequency domain. It is widely accepted that when dimension of input features increases, classification and generalization performances degrade. High dimensionality is expected to be transferred into vectors occupying a space of lower dimension using a feature selection or reduction approach $^{(6,8-10)}$.

The authors have attempted to propose an effective dimension reduction method for EMG motion classification using TF features. A simple classification method has been developed based on frequency division ${ }^{(8,10)}$. This method separates the frequency domain into four sub-bands by shape characters of the spectrum, and uses mean power level of each sub-band as indicator of spectral changes. Classification of six forearm motions was achieved using this method on EMG TF features extracted with STFT.

In this paper, the authors extended the frequency-division method and applied it to the wavelet-based TF representation for dimension reduction.
To validate the proposed method, experiments with four subjects and eleven forearm motions have been conducted. An MLP classifier was used to classify the forearm motions, including seven single motions and four simultaneous motions, based on TF features of six-channel EMG signals. This paper studied the average overall classification accuracies and the confusion matrices of classification results of eleven motions. Beside the classification performance, the number of sub-bands was also investigated with the replacing feature dimension of each channel set as two, four, and six.

\section{Methods}

\subsection{Experimental Configuration}

Four intact subjects (A, B, C, and D; all male) participated in this study. All subjects gave their informed consent before participation.

For EMG signal acquisition, six pairs of $\mathrm{Ag} / \mathrm{AgCl}$ surface electrodes (NT-511G, NIHON KODEN Corp.) were attached on the subjects' dominant forearm, as indicated in Fig. 1. The EMG signals were recorded in bipolar derivations, and a reference electrode was placed on a bony prominence at the elbow of the same side. A maximal voluntary contraction (MVC) method was applied in the pre-processing stage in order to correct differences in activation levels between channels. A second-order Butterworth band-pass filter, with cut-off frequencies as 5.3 and $250 \mathrm{~Hz}$, was applied to the raw EMG signals. The EMG signals were amplified and recorded with a multi-telemeter system (WEB-9500, NIHON KODEN Corp.), with the sampling frequency as $1 \mathrm{kHz}$. The motion classification experiments were conducted in an off-line manner.

The experiments focused on an 11-motion problem involving hand and wrist motions. The eleven motions were: hand grasping (GR), hand opening (OP), wrist

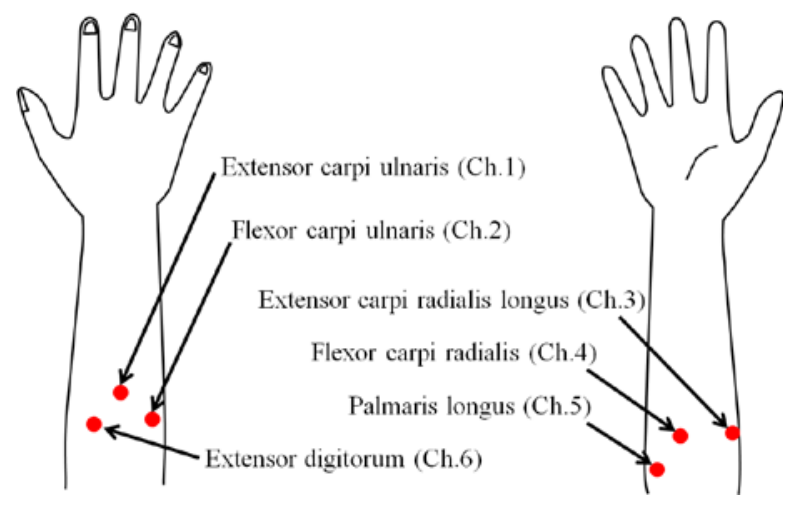

Fig. 1 Six EMG channels for signal acquisition. 


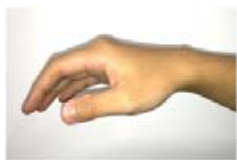

Neutral

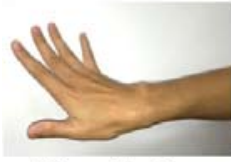

Ulnar flexion

(UF)

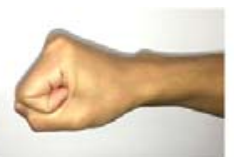

Grasp (GR)

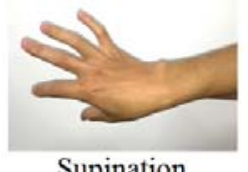

(SU)

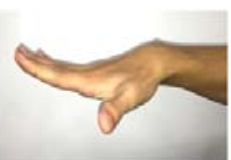

Open (OP)

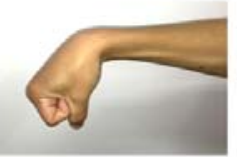

Grasp +

Wrist flexion

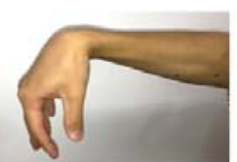

Wrist flexion

(WF)

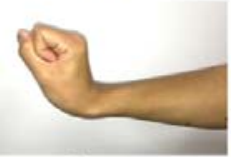

Grasp +

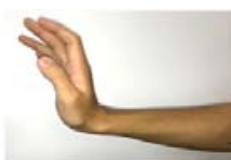

Wrist extension

(WE)

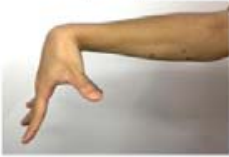

Open +

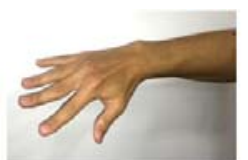

Radial flexion

(RF)

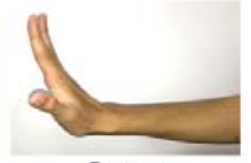

Open +

Fig. 2 Eleven forearm motions, including four simultaneous motions, considered in this study.

flexion (WF), wrist extension (WE), radial flexion (RF), ulnar flexion (UF), supination (SU), hand grasping + wrist flexion (GF), hand grasping + wrist extension (GE), hand opening + wrist flexion (OF), , and hand opening + wrist extension (OE). Figure 2 depicts examples of these motions. In each experimental session, the subject was instructed to perform these eleven motions twice in a predefined order. Each contraction was about $5 \mathrm{~s}$ in duration, with 3-5s relaxing periods (in the neutral position) between consecutive contractions.

\subsection{TF Representation with Wavelet Transform}

Raw EMG signals for the $n$th channel are defined as $E_{n}(n=1, \ldots, N)$. Time-frequency representation of $E_{n}$ can be obtained using continuous WT as follows,

$$
W_{n}(b, a)=\frac{1}{\sqrt{a}} \int E_{n}(t) \overline{\psi\left(\frac{t-b}{a}\right)} d t
$$

where $a$ is the scale parameter $(a=1, \ldots, A)$, which corresponds to the frequency information, $b$ stands for the time shift parameter $(b=1, \ldots, B)$, and $\psi(\cdot)$ is the mother
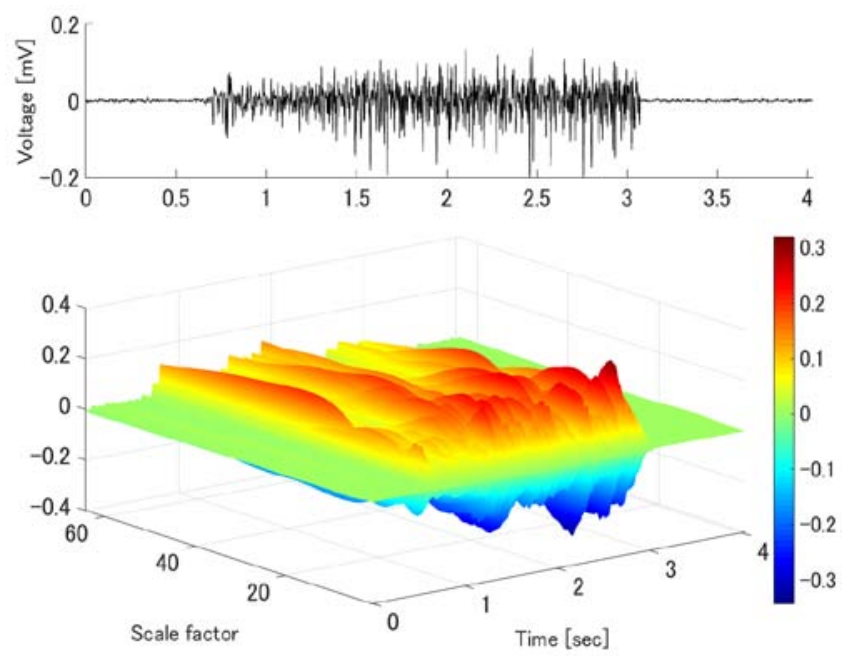

Fig. 3 Examples of raw EMG signals (upper panel) and wavelet coefficients with Coif4 MWF (lower panel). wavelet function (MWF). The parameter $B$ is the number of samples in the analyzed signal $\left(B=T \times f_{s}\right)$, where $T$ is the time length of raw EMG signals, and $f_{s}$ is the sampling frequency. Figure 3 shows an example of the wavelet coefficients calculated with MWF of Coiflets order 4 (Coif4). The wavelet coefficients consist of $A \times B$ elements.

For motion classification, the TF features can be extracted frame by frame along the timeline. At every time, dimension of the original feature set is quite large, say 384 for $A=64$. Such dimension size usually cause larger computational burden and more training epochs, so that may significantly affect classification performance. A compact frequency representation is needed to replace the original one.

\subsection{Dimension Reduction using Frequency Division}

This study extends the frequency-division method proposed in our previous study ${ }^{(8)}$ into a generalized form, and applies it to the wavelet-based TF representations of EMG signals. The frequency domain is divided into several sub-bands and the number of sub-bands is denoted as $S$. The division of sub-bands can be made with even spacing ${ }^{(9)}$ or by the shape characters of spectrum. Feature(s) of each sub-band can be defined with spectral indicators such as mean power, total power, and power spectrum ratio.

Since frequency representation of WT has uneven resolution over the bandwidth. A division with even spacing is not considered in this paper. With respect to the results of $\operatorname{Ref}^{(8)}$, the boundary frequencies are set as Table 1 for division of $S=2,4$, and 6 . The basic idea is that major

Table 1. Boundary frequencies on the bandwidth.

\begin{tabular}{|l|l|}
\hline$S$ & \multicolumn{1}{|c|}{ Boundary frequency $[\mathrm{Hz}]$} \\
\hline 2 & $5, \mathbf{8 0}, 250$ \\
\hline 4 & $5,40, \mathbf{8 0}, 150,250$ \\
\hline 6 & $5,40,60, \mathbf{8 0}, 120,150,250$ \\
\hline
\end{tabular}


characteristic frequency peaks can be found in the range of $40-150 \mathrm{~Hz}$, and this interval is further separated at $80 \mathrm{~Hz}$ in order keep discriminative information for motion classification ${ }^{(10)}$. First split the full bandwidth $5-250 \mathrm{~Hz}$ into lower and upper halves at $80 \mathrm{~Hz}$, then increase split points on both sides for the required number of sub-bands. In addition, the frequencies corresponding to the scale parameters differ on MWFs, the frequency-division is transformed to its scale-division counterpart, using the scale parameters closest to the boundary frequencies.

Mean power levels are then obtained at each sub-band for frames. Suppose the frame length is $L$ and the shift step between consecutive frames is $J$, the mean power for sub-band $s$ of the channel $n$ in the fame $k$ can be expressed as follows,

$$
P_{s, n}(k)=\frac{1}{C_{s} \cdot L} \sum_{a \in B_{S}} \sum_{(k-1) J}^{(k-1) J+L}\left|W_{n}(b, a)\right|
$$

where $B_{s}$ is the set of scales in the sub-band $s$, and $C_{s}$ is the number of elements in $B_{s}$. Absolute values of the wavelet coefficients are used.

These mean powers of sub-bands are combined to form the feature vector for each EMG channel,

$$
\alpha_{n}(k)=\left[P_{1, n}(k), \cdots, P_{S, n}(k)\right] .
$$

Summing up all $N$ channels, we have a novel feature vector for frame $k$ as

$$
\boldsymbol{X}(k)=\left[\alpha_{1}(k), \cdots, \alpha_{N}(k)\right] .
$$

Therefore the original feature vector of $A \times N$ dimensions is replaced by a compact one with dimension $D=S \times N$. This EMG feature $\boldsymbol{X}$ is input to an MLP to classify simultaneous motions on every frame.

\subsection{Simultaneous Motion Classification}

Motion classification is performed using an MLP classifier. In this study, MLP is trained with a traditional back-propagation method, which is a gradient descent based training algorithm. EMG data recorded in all experimental sessions are split into training and test data sets. During the train procedure, MLP is trained with ten sets of initial weights, which are randomly chosen. These trained MLPs then classify the test data so that ten sets classification results are obtained.

The output layer of MLP consists of $M$ nodes (one for a motion), and the $m$ th output is denoted as $O_{m}$. In order to prevent misclassification, entropy of classifications is calculated based on MLP's outputs. The outputs of MLP is in the interval $[0,1]$, and they are normalized in order to reach a probabilistic metric.

$$
\begin{gathered}
p_{m}(k)=\frac{O_{m}(k)}{\sum_{m^{\prime}=1}^{M} O_{m^{\prime}}(k)} \\
H(k)=-\sum_{m=1}^{M} p_{m}(k) \log p_{m}(k)
\end{gathered}
$$

where $p_{m}$ is the normalized output. If the entropy $H(k)$ is less than a predefined threshold $H_{\mathrm{d}}$, the motion with the largest normalized output $p$ is determined as the intended motion according to Bayes' decision rule. Otherwise, the determination is suspended.

\section{Results}

Experiments were conducted using Coif 4 as MWF in Eq. (1). The scale factor $a=64$, in case of Coif4, corresponds to frequency $10.87 \mathrm{~Hz}$. The upper limit of scale, $A$, was set as 64 . The frame length $L$ was 256 with a shifting step $J$ as 64 . In the entropy rule, the threshold $H_{\mathrm{d}}$ was defined as 1.0 for the 11-motion problem.

\subsection{Overall Classification Accuracy}

Overall classification accuracy, denoted as $C R_{\text {all }}$, is defined as the average of motion classification rates, $C R_{\mathrm{m}}$, as follows,

$$
\begin{gathered}
C R_{m}=\frac{C_{m}}{N_{m}-\mathrm{SUS}_{m}} \\
C R_{\text {all }}=\frac{1}{M} \sum_{m=1}^{M} C R_{m}
\end{gathered}
$$

where $C_{m}$ is the number of correctly classified motion $m$, $N_{m}$ is the total number of motion $m$, and $\operatorname{SUS}_{m}$ stands for number of the suspended classification in test data of motion $m$.

Overall classification rates were calculated for each $s$. Since ten sets of initial weights were used to train the MLP classifier, ten sets of classification results were also included to evaluate the overall classification performance. Table 2 gives the mean values and standard deviations (SD) of $C R_{\text {all }}$ for ten sets of results.

Table 2. Overall Classification Rates for Ten Initial Weights used in MLP Training [\%].

\begin{tabular}{|c|c|c|c|c|}
\hline$s$ & Subj. A & Subj. B & Subj. C & Subj. D \\
\hline 2 & $94.68 \pm 0.82$ & $97.76 \pm 0.47$ & $96.13 \pm 0.64$ & $92.74 \pm 0.58$ \\
\hline 4 & $93.26 \pm 1.14$ & $96.99 \pm 0.66$ & $96.05 \pm 0.54$ & $90.73 \pm 1.26$ \\
\hline 6 & $88.40 \pm 1.85$ & $90.09 \pm 2.43$ & $89.06 \pm 1.95$ & $79.18 \pm 2.52$ \\
\hline
\end{tabular}


The mean values of $C R_{\text {all }}$, for $s=2$ and 4 , exceed $90 \%$, and when $s=6$ three subjects' results are around 90\%. Generally high classification accuracy can be achieved with the proposed method. On the other hand, except $s$ of two and four in the case of Subject C, classification rates tend to decrease when $s$ increases. Significant difference $(p<0.01)$ can be found between each comparison pair. Classification results for motions and confusion matrices are investigated in the following sections.

\subsection{Classification Rates for Motions}

A summary of classification rates $(s=2)$ of each motion is listed in Table 3. Mean and SD values are calculated with ten sets of classification results.

Table 3. Classification Rates of 11 Motions $(s=2)$ [\%]

\begin{tabular}{|c|c|c|c|c|}
\hline$m$ & Subj. A & Subj. B & Subj. C & Subj. D \\
\hline GR & $94.5 \pm 4.4$ & $95.9 \pm 2.5$ & $98.2 \pm 1.2$ & $77.2 \pm 3.0$ \\
\hline OP & $82.0 \pm 1.6$ & $94.0 \pm 1.7$ & $96.1 \pm 3.2$ & $84.8 \pm 1.8$ \\
\hline WF & $99.2 \pm 0.8$ & $98.1 \pm 1.1$ & $94.6 \pm 0.9$ & $98.5 \pm 1.6$ \\
\hline WE & $95.5 \pm 2.3$ & $98.2 \pm 0.6$ & $92.6 \pm 1.4$ & $77.4 \pm 4.2$ \\
\hline RF & $99.7 \pm 0.6$ & $97.8 \pm 1.6$ & $95.9 \pm 1.5$ & $98.3 \pm 1.6$ \\
\hline UF & $99.6 \pm 0.5$ & $100.0 \pm 0.0$ & $98.4 \pm 0.9$ & $100.0 \pm 0.0$ \\
\hline PR & $100.0 \pm 0.0$ & $100.0 \pm 0.0$ & $100.0 \pm 0.0$ & $98.4 \pm 0.8$ \\
\hline GF & $84.5 \pm 4.9$ & $99.0 \pm 0.8$ & $97.0 \pm 1.4$ & $99.2 \pm 0.9$ \\
\hline GE & $98.0 \pm 2.7$ & $93.5 \pm 3.2$ & $98.3 \pm 1.4$ & $97.8 \pm 1.0$ \\
\hline OF & $94.2 \pm 3.3$ & $99.8 \pm 0.4$ & $97.6 \pm 1.3$ & $94.8 \pm 2.0$ \\
\hline OE & $94.2 \pm 0.7$ & $99.1 \pm 1.0$ & $88.9 \pm 2.2$ & $93.8 \pm 1.4$ \\
\hline
\end{tabular}

There is large variation among the classification rates. Comparing $C R_{\mathrm{m}} \mathrm{s}$ of simultaneous motions with those of signal motions, $C R_{\mathrm{m}} \mathrm{s}$ below $85 \%$ do not concentrate in the simultaneous group. Both simultaneous and single motions can be classified with the proposed method. In addition,
Table 4. Ranges of the $C R_{\mathrm{m}} \mathrm{s}$ [\%].

\begin{tabular}{|c|r|r|r|r|}
\hline s & \multicolumn{1}{|c|}{ Subj. A } & \multicolumn{1}{c|}{ Subj. B } & Subj. C & Subj. D \\
\hline 2 & 17.98 & 6.53 & 11.09 & 22.82 \\
\hline 4 & 18.55 & 12.43 & 9.06 & 30.50 \\
\hline 6 & 26.43 & 38.52 & 29.44 & 53.88 \\
\hline
\end{tabular}

ranges of the $C R_{\mathrm{m}} \mathrm{S}$, which are differences between the maximum and minimum $C R_{\mathrm{m}} \mathrm{s}$, are summarized in Table 4 for three conditions. Large ranges are found for $s=6$, and the ranges of subject $\mathrm{D}$ are larger than the others.

An example of the confusion matrix for classification results of one test trial (subject D) is depicted in Table 5. Misclassifications occur between simultaneous motion and its element motions, e.g. GF with GR or WF, confusion can also be found between simultaneous motions sharing same elements motions, e.g. GF with OF. On the other hand, misclassification between single motions, e.g. WF and WE, should be traced back to the training procedure of MLP.

\subsection{Training Procedure}

The MLP classifier consists of three layers. The input layer has $D$ nodes, one node for an element of the input feature vector. The hidden layer has 300 nodes and the output layer eleven nodes. The training procedure of MLP continues until the performance gradient falls below $10^{-8}$, where the learning rate is 0.01 . The maximum number of training epochs is 10,000 .

Training time for different $s$ is shown in Fig. 4. The mean and SD are obtained with training procedures using ten initial weights. All trainings terminate before training epoch number reaches its limit. Feature vectors of four sub-bands, i.e. 24 dimensions, cost the shortest training time. Since feature of $s=2$ provides half input size of four sub-bands division, the 11-motion classification may become difficult. On the other hand, features of six

Table 5. An example the confusion matrix of classification results (subj. D).

\begin{tabular}{|c|c|c|c|c|c|c|c|c|c|c|c|c|}
\hline & \multicolumn{11}{|c|}{ Actual } \\
\hline & & GR & $\mathrm{OP}$ & WF & WE & $\mathrm{RF}$ & UF & PR & GF & GE & OF & $\mathrm{OE}$ \\
\hline \multirow{12}{*}{ 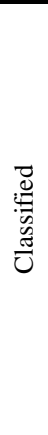 } & GR & 142 & 0 & 0 & 0 & 2 & 0 & 0 & 6 & 0 & 0 & 0 \\
\hline & OP & 0 & 122 & 0 & 0 & 0 & 0 & 2 & 0 & 0 & 0 & 3 \\
\hline & WF & 0 & 0 & 136 & 13 & 0 & 0 & 2 & 2 & 0 & 4 & 0 \\
\hline & WE & 0 & 0 & 0 & 104 & 0 & 0 & 0 & 1 & 2 & 0 & 1 \\
\hline & $\mathrm{RF}$ & 0 & 0 & 0 & 0 & 128 & 1 & 0 & 0 & 0 & 0 & 0 \\
\hline & UF & 0 & 0 & 0 & 0 & 0 & 139 & 0 & 0 & 0 & 0 & 1 \\
\hline & PR & 2 & 0 & 0 & 3 & 4 & 4 & 133 & 0 & 1 & 0 & 0 \\
\hline & GF & 12 & 0 & 0 & 0 & 0 & 0 & 1 & 125 & 0 & 2 & 0 \\
\hline & GE & 0 & 1 & 0 & 26 & 0 & 0 & 0 & 0 & 143 & 2 & 0 \\
\hline & $\mathrm{OF}$ & 0 & 2 & 1 & 0 & 0 & 0 & 7 & 3 & 0 & 120 & 0 \\
\hline & $\mathrm{OE}$ & 3 & 1 & 1 & 5 & 0 & 0 & 0 & 2 & 2 & 0 & 150 \\
\hline & $\begin{array}{l}\text { Sus. } \\
\text {. }\end{array}$ & $\bar{~} 12$ & $\bar{~} 17$ & $\bar{~} \overline{6}$ & $\bar{~} \overline{4}$ & $\bar{~} 15$ & $\overline{77}$ & 12 & $\bar{~} 12$ & $\overline{22}$ & 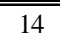 & 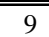 \\
\hline
\end{tabular}




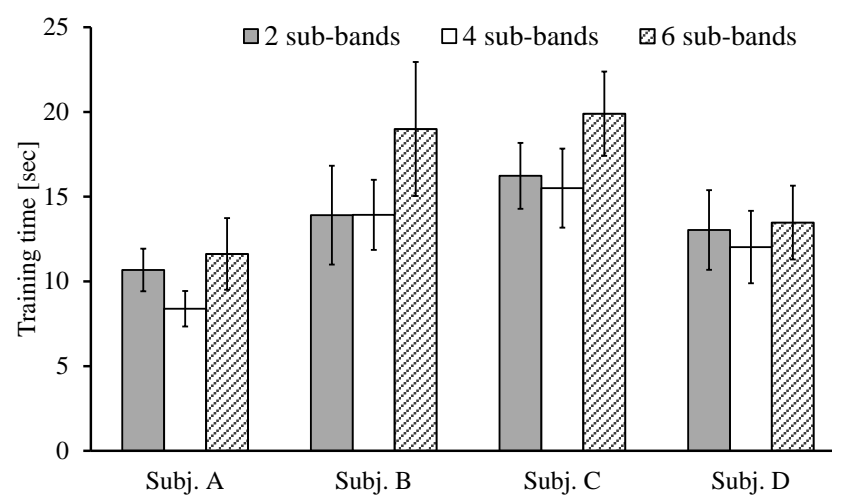

Fig. 4 Training time for different sub-band numbers.

sub-bands have 36 elements, the training maybe tend to hard to converge.

\section{Conclusions}

This paper attempts to achieve simultaneous motion classification using wavelet-based time-frequency features of EMG signals. In order to tackle the curse of dimensionality problem of $\mathrm{TF}$ features, the proposed method uses a frequency-division approach to reduce the elements in feature vectors. To examine the classification performance of the proposed method, motion classification experiments, including four simultaneous motions and seven single ones, have been carried out with four subjects. A traditional MLP classifier has been used for the 11-motion problem. Overall classification rates as well as classification results for each motion were investigated. In the experiments, both simultaneous and single motions were classified, and generally high overall classification rates were confirmed.

Ranges of classification rates indicate that quite large variance exits in the results of different band-division number for four subjects. Detailed check of the confusion matrices reveals that misclassification occurs not only between simultaneous motions with their component single motions but some single motions are misclassified as other single ones. In addition, it is found that training performance is largely related to the classification accuracy. This may be a clue for why classification rates fall when the division number increases. It should be mentioned that no sophisticated training algorithm was used to train the MLP classifier, also the number of nodes in hidden layer was fixed as 300. Further investigation on the training procedure may provide other evaluation of the frequency-division.

In the future research, we would like to improve the frequency-division process considering uneven spacing of the wavelet scales. Also, comparison studies with FT features extracted with STFT should be investigated.

\section{References}

(1) E. Scheme and K. Englehart: "Electromyogram pattern recognition for control of powered upper-limb prostheses: state of the art and challenges for clinical use”, J. Rehabil. Res. Dev., Vol. 48, pp. 643-659, 2011

(2) T. Tsuji, K. Shima, and Y. Murakami: "Pattern classification of combined motions based on muscle synergy theory”, J. Robot. Soc. Jpn., Vol. 28, No. 5, pp. 606-613, 2010

(3) N. Jiang, K. Englehart, and P. Parker: "Extracting simultaneous and proportional neural control information for multiple-DOF prostheses from the surface electromyographic signal”, IEEE Trans. Biomed. Eng., Vol. 56, No. 4, pp. 1070-1080, 2009

(4) A.J. Young, L.H. Smith, E.J. Rouse, and L.J. Hargrove: "Classification of simultaneous movements using surface EMG pattern recognition”, IEEE Trans. Biomed. Eng., Vol. 60, No. 5, pp. 1250-1258, 2013

(5) J. Ngeo, T. Tamei, and T. Shibata: “Continuous and simultaneous estimation of finger kinematics using inputs from an EMG-to-muscle activation model”, J. Neuroeng. Rehabil. Vol. 11, 122, 2014

(6) K. Englehart, B. Hudgins, P.A. Parker, M. Stevenson: "Classification of the myoelectric signal using time-frequency based representations”, Med. Eng. Phys., Vol. 21, pp. 431-438, 1999

(7) S. Karlsson, J. Yu, and M. Akay: "Time-frequency analysis of myoelectric signals during dynamic contractions: a comparative study", IEEE Trans. Biomed. Eng., Vol. 47, No. 2, pp. 228-238, 2000

(8) T. Mukaeda and N. Bu: "Forearm motion classification based on frequency characteristics of EMG signals", Proc. of the 4th Intern. Symp. on Technology for Sustainability, Paper-ID 071, 2014

(9) A.-C. Tsai, T.-H. Hsieh, J.-J. Luh, and T.-T. Lin: “A comparison of upper-limb motion pattern recognition using EMG signals during dynamic and isometric muscle contractions”, Biomed. Signal Process. Control, Vol. 11, pp. 17-26, 2014

(10)T. Mukaeda, N. Bu, K. Shibasato, H. Ohtsuka: "Forearm motion classification based on time-frequency characteristics of EMG signals”, Trans. on GIGAKU, 2016. (in Press) 